CONFORMAL GEOMETRY AND DYNAMICS

An Electronic Journal of the American Mathematical Society

Volume 11, Pages 44-55 (March 15, 2007)

S $1088-4173(07) 00156-7$

\title{
FREE SUBGROUPS OF SURFACE MAPPING CLASS GROUPS
}

\author{
JAMES W. ANDERSON, JAVIER ARAMAYONA, AND KENNETH J. SHACKLETON
}

\begin{abstract}
We quantify the generation of free subgroups of surface mapping class groups by pseudo-Anosov mapping classes in terms of their translation distance and the distance between their axes in Teichmüller's metric. The method makes reference to Teichmüller space only.
\end{abstract}

\section{INTRODUCTION}

Free subgroups of mapping class groups have attracted considerable attention from a number of authors over a number of years. It is a classical result, proven independently and simultaneously by Ivanov [11] and by McCarthy [16], that any collection $\mathcal{A}$ of pseudo-Anosov mapping classes with pairwise distinct axes freely generates a free group of rank $|\mathcal{A}|$, so long as each element is first raised to a sufficiently high power. The supporting argument is based on the so-called PingPong Lemma, quoted here in Section 4 , and makes reference to Thurston's boundary of Teichmüller space.

The purpose of this work is to establish a ping-pong argument entirely inside Teichmüller space. This allows us to not only recover the result of Ivanov and of McCarthy by somewhat different means, but also to use the Teichmüller metric to quantify sufficiently high powers in a natural way. Our work relies on an application of Minsky's Bounded Projection Theorem [19. One may also use Thurston's train tracks to quantify sufficiently high powers; see Hamidi-Tehrani 8$]$.

The plan of this paper is as follows. In Section 2 we introduce all the background and notation we shall need. In Section 3 we recover the known result that axes of independent pseudo-Anosov mapping classes cannot be asymptotic. In Section 4 we exhibit ping-pong sets inside Teichmüller space for independent pseudo-Anosov mapping classes and use these to show that sufficiently high powers of independent pseudo-Anosov mapping classes generate a free group. In Section 5 we give a quantitative version of this result, with Theorem 5.7 our aim. Sections 4 and 5 are logically independent, but both rest firmly on Sections 2 and 3 .

\section{BACKGROUND}

We refer the reader to [2, 9] and [12] for detailed studies of geodesic and measured geodesic laminations, Teichmüller spaces and mapping class groups, respectively, and recall only what we need here. Throughout this paper, a surface $\Sigma$ will

Received by the editors May 152006 and, in revised form, November 8, 2006.

2000 Mathematics Subject Classification. Primary 20F65; Secondary 57M50.

The third author was partially supported by a short-term Japan Society for the Promotion of Science post-doctoral fellowship for foreign researchers, number PE05043.

(C)2007 American Mathematical Society Reverts to public domain 28 years from publication 
mean an orientable connected surface of negative Euler characteristic, with genus $g$ and $p$ punctures, and with empty boundary. A curve on $\Sigma$ is the free homotopy class of a simple closed loop that is neither homotopic to a point nor to a puncture, and we denote by $\mathcal{S}(\Sigma)$ the set of all curves on $\Sigma$. We say that two curves are disjoint if they can be realised disjointly, and if they are not disjoint we say they intersect essentially or are transverse.

2.1. Teichm̈uller space. The Teichmüller space $T(\Sigma)$ is the space of all marked finite area hyperbolic structures on $\Sigma$, up to homotopy. More specifically, a point in $T(\Sigma)$ is an equivalence class $[(\sigma, f)]$, where $\sigma$ is a finite area hyperbolic structure on $\Sigma$ and $f: \Sigma \longrightarrow \sigma$ is a homeomorphism, called a marking of $\sigma$. One declares two pairs $(\sigma, f)$ and $\left(\sigma^{\prime}, g\right)$ to be equivalent if and only if $g \circ f^{-1}$ is homotopic to an isometry between $\sigma$ and $\sigma^{\prime}$. To simplify our notation, we will use the same symbol to denote a point in $T(\Sigma)$ as to denote a particular marked finite area hyperbolic structure on $\Sigma$.

The space $T(\Sigma)$ is homeomorphic to an open ball of dimension $6 g-6+2 p$, and can be compactified by attaching the space $\operatorname{PML}(\Sigma)$ of all projective measured laminations on $\Sigma$, topologically a sphere of dimension $6 g-7+2 p$. The closed ball $T(\Sigma) \cup \operatorname{PML}(\Sigma)$ is sometimes known as the Thurston compactification of Teichmüller space, and we shall denote it by $\overline{T(\Sigma)}$.

Any curve $\alpha \in \mathcal{S}(\Sigma)$ induces a length function $\ell_{\alpha}$ on $T(\Sigma)$, where $\ell_{\alpha}(x)$ denotes the length of the unique geodesic representative of the class $\alpha$ in the hyperbolic structure $x$ on $\Sigma$. Given $\epsilon>0$, the $\epsilon$-thick part of Teichmüller space is defined by

$$
T_{\geq \epsilon}(\Sigma)=\left\{x \in T(\Sigma): \ell_{\alpha}(x) \geq \epsilon \text { for all } \alpha \in \mathcal{S}(\Sigma)\right\}
$$

and the $\epsilon$-thin part of Teichmüller space is defined by

$$
T_{\leq \epsilon}(\Sigma)=\left\{x \in T(\Sigma): \ell_{\alpha}(x) \leq \epsilon \text { for some } \alpha \in \mathcal{S}(\Sigma)\right\} .
$$

The space $T(\Sigma)$ admits two natural metrics, the Teichmüller metric and the WeilPetersson metric. In this paper we will only consider the Teichmüller metric, and refer the reader to 25] for a thorough study of the Weil-Petersson geometry. Given points $x, y \in T(\Sigma)$, the Teichmüller distance between $x=[(\sigma, f)]$ and $y=\left[\left(\sigma^{\prime}, g\right)\right]$ is defined as

$$
\mathrm{d}_{T}(x, y)=\frac{1}{2} \inf \{\log (K(h))\},
$$

where the infimum ranges over all the quasiconformal homeomorphisms $h$ in the homotopy class of $f \circ g^{-1}$ and $K(h)$ is the dilatation of $h$. By a celebrated result of Teichmüller, for any two points in Teichmüller space there is a unique quasiconformal homeomorphism $h$ (in the appropriate homotopy class) realising their distance. Endowed with the Teichmüller metric, $T(\Sigma)$ is a uniquely geodesic, proper metric space. It is worth noting, however, the Teichmüller metric is not non-positively curved in any standard sense (see [14, 15, [18, and [3]).

For subsets $X$ and $Y$ of $T(\Sigma)$, we define the nearest point distance $\mathrm{d}_{T}(X, Y)$ to be

$$
\mathrm{d}_{T}(X, Y)=\inf \left\{\mathrm{d}_{T}(x, y): x \in X, y \in Y\right\} .
$$

2.2. Mapping class groups. The mapping class group $\operatorname{MCG}(\Sigma)$ of $\Sigma$ is the group of all homotopy classes of orientation preserving self-homeomorphisms of $\Sigma$. It is a finitely presented group, generated by a finite collection of Dehn twists about simple closed curves of $\Sigma$. There is a natural action of $\operatorname{MCG}(\Sigma)$ on $T(\Sigma)$ by changing the 
marking; the quotient $T(\Sigma) / \mathrm{MCG}(\Sigma)$ is the moduli space of $\Sigma$. Except for a few low-dimensional cases, the mapping class group $\operatorname{MCG}(\Sigma)$ is isomorphic to an index 2 subgroup of the full isometry group of both the Teichmüller metric and the WeilPetersson metric, by results of Royden [22] and Masur-Wolf [15], respectively.

There are some similarities between the action of $\operatorname{MCG}(\Sigma)$ on $T(\Sigma)$, equipped with the Teichmüller metric, and that of a geometrically finite group acting isometrically on a simply-connected Hadamard manifold of pinched negative curvature. For example, the elements of $\operatorname{MCG}(\Sigma)$ can be classified in a manner that mimics the classification of the isometries of the pinched Hadamard manifold according to their dynamics on its ideal boundary. An infinite order mapping class is either reducible, so it fixes some non-empty and finite collection of disjoint curves, or is otherwise pseudo-Anosov, and fixes exactly two ideal points.

A pseudo-Anosov mapping class $\phi$ is represented by a pseudo-Anosov diffeomorphism $\Sigma$. That is, there exists a real number $r=r(\phi)>1$, the dilatation of $\phi$, such that for any hyperbolic metric $(\Sigma, \sigma)$ there exists a unique diffeomorphism $f$ representing $\phi$ and two measured laminations, $\lambda^{-}$and $\lambda^{+}$, geodesic in $\sigma$ such that $f\left(\lambda^{+}\right)=r \lambda^{+}$and $f\left(\lambda^{-}\right)=\frac{1}{r} \lambda^{-}$. We shall write $\lambda^{ \pm}$to denote either element of the set $\left\{\lambda^{+}, \lambda^{-}\right\}$. The measured lamination $\lambda^{ \pm}$satisfies the following three fundamental properties 23:

(1) $\lambda^{ \pm}$is uniquely ergodic: If $\mu$ is a measured lamination whose $\operatorname{support} \operatorname{supp}(\mu)$ is equal to $\operatorname{supp}\left(\lambda^{ \pm}\right)$, then $\mu$ and $\lambda^{ \pm}$are proportional.

(2) $\lambda^{ \pm}$is minimal: If $\mu$ is a measured lamination satisfying $\operatorname{supp}(\mu) \subseteq \operatorname{supp}\left(\lambda^{ \pm}\right)$, then either $\operatorname{supp}(\mu)=\emptyset$ or $\operatorname{supp}(\mu)=\operatorname{supp}\left(\lambda^{ \pm}\right)$.

(3) $\lambda^{ \pm}$is maximal: If $\mu$ is a measured lamination satisfying $\operatorname{supp}\left(\lambda^{ \pm}\right) \subseteq \operatorname{supp}(\mu)$, then $\operatorname{supp}(\mu)=\operatorname{supp}\left(\lambda^{ \pm}\right)$.

We will say a projective measured lamination is uniquely ergodic if one (and hence any) representative of its projective class is a uniquely ergodic measured lamination, and whenever $\lambda$ is uniquely ergodic we shall also use, where there can be no ambiguity, $\lambda$ to denote both a measured lamination and its projective class.

The fixed point set $\operatorname{Fix}(\phi)$ of $\phi$ in $\overline{T(\Sigma)}$ is precisely $\left\{\lambda^{+}, \lambda^{-}\right\}$. These fixed points behave like attracting and repelling fixed points for $\phi$. More specifically, with $s \in\{1,-1\}$, for any neighbourhood $U$ of $\lambda^{s}$ in $\overline{T(\Sigma)}$ and any compact set $K$ in $\overline{T(\Sigma)} \backslash\left\{\lambda^{-s}\right\}$ we have $\phi^{s n}(K) \subseteq U$ for sufficiently large $n$ (see [12]). It is known that a pseudo-Anosov mapping class $\phi$ fixes a bi-infinite Teichmüller geodesic, the axis of $\phi$, on which it acts by translation. By the above discussion, the set of accumulation points of this axis on $\operatorname{PML}(\Sigma)$ is $\operatorname{Fix}(\phi)=\left\{\lambda^{+}, \lambda^{-}\right\}$.

The translation distance $\operatorname{Tr}(\phi)=\inf \left\{\mathrm{d}_{T}(x, \phi(x)): x \in T(\Sigma)\right\}$ of a pseudoAnosov mapping class is always realised, and is always realised on the axis of $\phi$. Furthermore, both Tr and the property of being pseudo-Anosov are invariant under conjugation. The following result is due to Ivanov [10].

Theorem 2.1 (10]). For a surface $\Sigma$ and $L>0$, there are only finitely many conjugacy classes of pseudo-Anosov mapping classes of translation distance at most L.

It follows that there exists a constant $\ell_{\min }=\ell_{\min }(\Sigma)>0$ such that all pseudoAnosov mapping classes in $\operatorname{MCG}(\Sigma)$ have translation distance at least $\ell_{\text {min }}$. Lower bounds for $\ell_{\min }$, in terms of the topological type of $\Sigma$, have been found by Penner [20]. 
The following terminology is due to Minsky [19].

Definition 2.2. For a surface $\Sigma$ and $\epsilon>0$, a Teichmüller geodesic $c$ is said to be $\epsilon$-precompact if $c$ is entirely contained in the $\epsilon$-thick part $T_{\geq \epsilon}(\Sigma)$ of $T(\Sigma)$.

For any pseudo-Anosov mapping class $\phi$, the projection of its axis into moduli space is compact. Moreover, by the continuity of the length functions $x \longrightarrow \ell_{\alpha}(x)$, there is a uniform lower bound on all $\ell_{\alpha}(x)$, where $\alpha \in \mathcal{S}(\Sigma)$ and $x$ lies on the axis of $\phi$. Applying Theorem 2.1 to remove all dependence on $\phi$ yields the following.

Corollary 2.3. For a surface $\Sigma$ and $L>0$, there exists a positive real number $\epsilon=\epsilon(L, \Sigma)$ such that, if $\phi$ is any pseudo-Anosov mapping class of translation distance at most $L$, the axis of $\phi$ is e-precompact.

Let $\phi, \psi \in \operatorname{MCG}(\Sigma)$ be two pseudo-Anosov mapping classes and let $\operatorname{Fix}(\phi)$ and $\operatorname{Fix}(\psi)$ be their respective fixed point sets in $\overline{T(\Sigma)}$. It is known (see [17]) that either $\operatorname{Fix}(\phi)=\operatorname{Fix}(\psi)$, in which case $\phi$ and $\psi$ have non-zero powers which are powers of the same pseudo-Anosov mapping class, or $\operatorname{Fix}(\phi) \cap \operatorname{Fix}(\psi)=\emptyset$. This prompted the following definition.

Definition 2.4 ([17]). For a surface $\Sigma$, two pseudo-Anosov mapping classes $\phi, \psi \in$ $\operatorname{MCG}(\Sigma)$ are said to be independent if $\operatorname{Fix}(\phi) \cap \operatorname{Fix}(\psi)=\emptyset$.

\section{Divergence of PSEudo-Anosov axes}

It is one consequence of Minsky's Bounded Projection Theorem [19, found by Farb-Mosher [7, that the axes of two independent pseudo-Anosov mapping classes cannot be asymptotic in $T(\Sigma)$; that is, their Hausdorff distance is not finite. We offer a proof of a very much related result, namely that the distance function between the axes of independent pseudo-Anosov mapping classes is a proper function. (The corresponding result for the Weil-Petersson metric is due to DaskalopoulosWentworth [6].) We shall not need the Bounded Projection Theorem here, but instead a theorem of Wolpert [24] and a special case of Lemma 2.1 from [5].

Theorem 3.1 ([24]). For a surface $\Sigma$ and $D>0$, let $x, y \in T(\Sigma)$ with $\mathrm{d}_{T}(x, y) \leq$ $D$. Then, for any curve $\alpha \in \mathcal{S}(\Sigma)$, we have

$$
e^{-2 D} \ell_{\alpha}(x) \leq l_{\alpha}(y) \leq e^{2 D} \ell_{\alpha}(x) .
$$

Although the intersection number of two arbitrary projective measured laminations is not well defined, we can still decide whether their intersection number should be zero or non-zero.

Lemma $3.2([5])$. For a surface $\Sigma$, let $\lambda, \lambda^{\prime} \in \operatorname{PML}(\Sigma)$. Let $\left(x_{n}\right)$ be a sequence of points in $T(\Sigma)$ converging to $\lambda$ and let $\left(\alpha_{n}\right)$ be a sequence of curves converging to $\lambda^{\prime}$ as measured laminations. Suppose there exists $R>0$ such that $\ell_{\alpha_{n}}\left(x_{n}\right) \leq R$. Then, $i\left(\lambda, \lambda^{\prime}\right)=0$.

We are now ready to prove the distance function restricted to the axes of a pair of pseudo-Anosov mapping classes is a proper map. Recall, a map is said to be proper if the preimage of any compact subset of the range is compact.

Proposition 3.3. For a surface $\Sigma$, let $\phi, \psi \in \mathrm{MCG}(\Sigma)$ be two independent pseudoAnosov mapping classes and let $c, c^{\prime}: \mathbb{R} \longrightarrow T(\Sigma)$ be arc-length parametrizations of their respective axes. Then, the map $(t, s) \longrightarrow \mathrm{d}_{T}\left(c(t), c^{\prime}(s)\right)$ is a proper map. 
Proof. Suppose the result is not true. Then, there are unbounded sequences $\left(t_{n}\right)$ and $\left(s_{n}\right)$ in $\mathbb{R}$ and a real number $M>0$ such that $\mathrm{d}_{T}\left(c\left(t_{n}\right), c^{\prime}\left(s_{n}\right)\right) \leq M$ for all $n \in \mathbb{N}$. By passing to subsequences if need be, we may assume that $\left(t_{n}\right)$ and $\left(s_{n}\right)$ are each either monotonically increasing or monotonically decreasing. Let us suppose they are both monotonically increasing, as the remaining cases can be treated analogously.

Let $\lambda, \mu \in \operatorname{PML}(\Sigma)$ be such that $x_{n}=c\left(t_{n}\right) \longrightarrow \lambda$ and $y_{n}=c^{\prime}\left(s_{n}\right) \longrightarrow \mu$ in $\overline{T(\Sigma)}$, as $n \longrightarrow \infty$. Then, for all $n \in \mathbb{N}$, there exists $x_{n}^{\prime} \in c(\mathbb{R})$ such that $\mathrm{d}_{T}\left(x_{n}, x_{n}^{\prime}\right) \leq$ $\operatorname{Tr}(\phi)$ and $x_{n}^{\prime}=\phi^{k_{n}}\left(x_{0}\right)$, for some $x_{0} \in c$ and $k_{n}=k(n)$. In particular, we have $k_{n} \longrightarrow \infty$ as $n \longrightarrow \infty$. Since $\mathrm{d}_{T}\left(x_{n}, y_{n}\right) \leq M$, we see that $\mathrm{d}_{T}\left(x_{n}^{\prime}, y_{n}\right) \leq M+\operatorname{Tr}(\phi)$. Denote by $M^{\prime}$ the upper bound $M+\operatorname{Tr}(\phi)$.

Choose any curve $\alpha \in \mathcal{S}(\Sigma)$ and let $\alpha_{n}=\phi^{k_{n}}(\alpha)$, noting $\left(\alpha_{n}\right)$ converges to $\lambda$ as measured laminations. It follows from the definition of the action of MCG $(\Sigma)$ on $T(\Sigma)$ that $\ell_{\alpha_{n}}\left(x_{n}^{\prime}\right)=\ell_{\alpha}\left(x_{0}\right)$. This fact, together with the upper bound in Theorem 3.1, implies $\ell_{\alpha_{n}}\left(y_{n}\right) \leq e^{2 M^{\prime}} \ell_{\alpha}\left(x_{0}\right)$. Therefore, the sequences $\left(y_{n}\right)$ and $\left(\alpha_{n}\right)$ satisfy the hypotheses of Lemma 3.2 , with $R$ equal to $e^{2 M^{\prime}} \ell_{\alpha}\left(x_{0}\right)$, and we conclude $i(\lambda, \mu)=0$. As $\lambda$ and $\mu$ are both maximal and both minimal, we find $\operatorname{supp}(\lambda)=\operatorname{supp}(\mu)$ and thus $\lambda=\mu$, since $\lambda$ and $\mu$ are uniquely ergodic. However, this is contrary to the assumption that $\phi$ and $\psi$ be independent as pseudo-Anosov mapping classes.

An immediate consequence of Proposition 3.3 is the following.

Corollary $3.4([7])$. For a surface $\Sigma$, let $\phi$ and $\psi$ be two independent pseudoAnosov mapping classes and let $c, c^{\prime}: \mathbb{R} \longrightarrow T(\Sigma)$ be arc-length parametrizations of their respective axes. Then, the Hausdorff distance between $c(\mathbb{R})$ and $c^{\prime}(\mathbb{R})$ is infinite.

\section{Ping-Ponging in Teichmüller space}

The purpose of this section is to exhibit ping-pong sets inside Teichmüller space for any given finite family of independent pseudo-Anosov mapping classes. This recovers the theorem of Ivanov [11] and McCarthy [16]. In Section 5 we shall give a quantitative version of this result.

For a closed subset $C$ of $T(\Sigma)$ and a point $x \in T(\Sigma)$, one defines the closest-point projection of $x$ into $C$ as

$$
\pi_{C}(x)=\left\{y \in C: \mathrm{d}_{T}(x, y) \leq \mathrm{d}_{T}(x, z) \text { for all } z \in C\right\} .
$$

Note $\pi_{C}(x)$ is non-empty since $T(\Sigma)$ is a proper metric space. For another subset $C^{\prime} \subset T(\Sigma)$, we define

$$
\pi_{C}\left(C^{\prime}\right)=\bigcup_{x \in C^{\prime}} \pi_{C}(x)
$$

Given any path $c: \mathbb{R} \longrightarrow T(\Sigma)$ we shall also use $\pi_{c}$ to denote $\pi_{c(\mathbb{R})}$, the projection to the image of $c$. The next result is Minsky's Bounded Projection Theorem, a union of Contraction Theorem (1), Corollary 4.1 and Theorem 4.2 from [19], and highlights some of the hyperbolic behaviour of any thick part of Teichmüller space. For its statement, we shall first need the following standard definition. 
Definition 4.1. Let $\left(X, d_{X}\right)$ and $\left(Y, d_{Y}\right)$ be metric spaces, and let $K \geq 1, \kappa \geq 0$. A $(K, \kappa)$-quasi-isometric embedding of $X$ into $Y$ is a map $f: X \longrightarrow Y$ such that

$$
\frac{1}{K} d_{X}\left(x, x^{\prime}\right)-\kappa \leq d_{Y}\left(f(x), f\left(x^{\prime}\right)\right) \leq K d_{X}\left(x, x^{\prime}\right)+\kappa,
$$

for all $x, x^{\prime} \in X$. A $(K, \kappa)$-quasi-geodesic in $Y$ is a $(K, \kappa)$-quasi-isometric embedding of a closed subinterval of $\mathbb{R}$ into $Y$.

Note, a quasi-geodesic need not be continuous. In what follows, $B_{r}(x)$ denotes the compact ball in $T(\Sigma)$ of radius $r$ and centre $x$.

Theorem $4.2([19])$. For a surface $\Sigma$ and $\epsilon>0$, there exists a constant $b=b(\epsilon, \Sigma)$ such that the following hold.

(1) Given any $\epsilon$-precompact geodesic $c$ and any $x \in T(\Sigma)$, we have

$$
\operatorname{diam}\left(\pi_{c}\left(B_{\mathrm{d}_{T}(x, c(\mathbb{R}))}(x)\right)\right) \leq b .
$$

(2) Given any $\epsilon$-precompact geodesic $c$ and any $x, y \in T(\Sigma)$, we have

$$
\operatorname{diam}\left(\pi_{c}(x) \cup \pi_{c}(y)\right) \leq \mathrm{d}_{T}(x, y)+4 b .
$$

(3) Given $K \geq 1$ and $\kappa \geq 0$, there exists a non-negative real number $M=$ $M(K, \kappa, \epsilon, \Sigma)$ such that the following holds: If $q$ is a $(K, \kappa)$-quasi-geodesic path in $T(\Sigma)$ whose endpoints are connected by an $\epsilon$-precompact Teichmüller geodesic $c$, then the image of $q$ is contained in the closed $M$-neighbourhood of the image of $c$.

The next result is a key ingredient for the ping-pong argument. Roughly speaking, it says precompact Teichmüller geodesics diverge "sufficiently fast". We shall make use of the following notation: Given an embedding $c: \mathbb{R} \longrightarrow T(\Sigma)$ and two of its points $x=c(t), x^{\prime}=c\left(t^{\prime}\right)$, we will write $x<x^{\prime}$ if $t<t^{\prime}$.

Proposition 4.3. For a surface $\Sigma$ and $\epsilon>0$, let $c, c^{\prime}: \mathbb{R} \longrightarrow T(\Sigma)$ be arc-length parametrizations of two $\epsilon$-precompact Teichmüller geodesics so that $O=c(0)$ and $O^{\prime}=c^{\prime}(0)$ realise the nearest point distance $D$ between $c(\mathbb{R})$ and $c^{\prime}(\mathbb{R})$. Then, there exist two points $P^{+}, P^{-} \in c(\mathbb{R})$ and two points $Q^{+}, Q^{-} \in c^{\prime}(\mathbb{R})$, with $P^{-}<O<P^{+}$ and $Q^{-}<O^{\prime}<Q^{+}$, such that the following hold:

(1) For all $x \in c(\mathbb{R})$ with $x>P^{+}$and all $y \in c^{\prime}(\mathbb{R})$ with $y>Q^{+}$,

$$
\mathrm{d}_{T}(x, y)>\max \left\{\mathrm{d}_{T}(O, x), \mathrm{d}_{T}\left(O^{\prime}, y\right)\right\} .
$$

(2) For all $x \in c(\mathbb{R})$ with $x<P^{-}$and all $y \in c^{\prime}(\mathbb{R})$ with $y<Q^{-}$,

$$
\mathrm{d}_{T}(x, y)>\max \left\{\mathrm{d}_{T}(O, x), \mathrm{d}_{T}\left(O^{\prime}, y\right)\right\} .
$$

Proof. We show only the first part of the proposition, as the second part follows by an analogous argument. Suppose, for contradiction, the statement is not true. Then, we can find two unbounded sequences $\left(x_{n}\right)$ and $\left(y_{n}\right)$ of points in $c(\mathbb{R})$ and $c^{\prime}(\mathbb{R})$, respectively, such that $O<x_{n}<x_{n+1}, O^{\prime}<y_{n}<y_{n+1}$ and $\mathrm{d}_{T}\left(x_{n}, y_{n}\right) \leq$ $\max \left\{\mathrm{d}_{T}\left(O, x_{n}\right), \mathrm{d}_{T}\left(O^{\prime}, y_{n}\right)\right\}$ for all $n \in \mathbb{N}$. Passing to a further subsequence if need be, we have $\mathrm{d}_{T}\left(O, x_{n}\right) \geq \mathrm{d}_{T}\left(O^{\prime}, y_{n}\right)$ for all $n$, or we have $\mathrm{d}_{T}\left(O, x_{n}\right) \leq \mathrm{d}_{T}\left(O^{\prime}, y_{n}\right)$ for all $n$. Without loss of generality, let us suppose the former holds.

Let $g_{n}$ be the $c(\mathbb{R})$-segment between $O$ and $x_{n}$, noting the length of $g_{n}$ tends to infinity as $n \longrightarrow \infty$. Let $q_{n}$ be the concatenation of the unique Teichmüller 
geodesic segment from $O$ to $O^{\prime}$, the $c^{\prime}(\mathbb{R})$-segment from $O^{\prime}$ to $y_{n}$, and the unique Teichmüller geodesic segment from $y_{n}$ to $x_{n}$. Then,

$$
\begin{aligned}
\operatorname{length}\left(q_{n}\right) & \leq D+\mathrm{d}_{T}\left(O^{\prime}, y_{n}\right)+\mathrm{d}_{T}\left(x_{n}, y_{n}\right) \\
& \leq D+\mathrm{d}_{T}\left(O, x_{n}\right)+\mathrm{d}_{T}\left(O, x_{n}\right)=D+2 \operatorname{length}\left(g_{n}\right) .
\end{aligned}
$$

Moreover,

$$
\operatorname{length}\left(g_{n}\right)=\mathrm{d}_{T}\left(O, x_{n}\right) \leq \mathrm{d}_{T}\left(O, O^{\prime}\right)+\mathrm{d}_{T}\left(O^{\prime}, y_{n}\right)+\mathrm{d}_{T}\left(y_{n}, x_{n}\right)=\operatorname{length}\left(q_{n}\right) .
$$

We deduce

$$
\text { length }\left(g_{n}\right) \leq \operatorname{length}\left(q_{n}\right) \leq D+2 \operatorname{length}\left(g_{n}\right),
$$

and therefore $q_{n}$ is a $(2, D)$-quasi-geodesic. In particular, notice that the constants of quasi-geodesicity are independent of $n$. By Theorem $4.2(3)$, there is a constant $M=M(2, D, \epsilon, \Sigma)$ such that the image of $q_{n}$ is contained in the closed $M$-neighbourhood of the image of $g_{n}$, for all $n \in \mathbb{N}$. Since $\mathrm{d}_{T}\left(O^{\prime}, y_{n}\right)$ tends to infinity as $n \longrightarrow \infty$, this implies that the Hausdorff distance between $c([0, \infty))$ and $c^{\prime}([0, \infty))$ is at most $M$. According to Proposition 3.3, this is a contradiction.

Corollary 4.4. For a surface $\Sigma$ and $\epsilon>0$, let $c, c^{\prime}: \mathbb{R} \longrightarrow T(\Sigma)$ be arc-length parametrizations of two $\epsilon$-precompact Teichmüller geodesics. Then, the set $\pi_{c^{\prime}}(c(\mathbb{R}))$ is bounded.

Proof. Let $D$ be the nearest-point distance between $c(\mathbb{R})$ and $c^{\prime}(\mathbb{R})$, and choose $O \in c(\mathbb{R})$ and $O^{\prime} \in c^{\prime}(\mathbb{R})$ such that $\mathrm{d}_{T}\left(O, O^{\prime}\right)=D$. Note $O^{\prime} \in \pi_{c^{\prime}}(O)$ and $O \in \pi_{c}\left(O^{\prime}\right)$. Suppose, for contradiction, that $\pi_{c^{\prime}}(c(\mathbb{R}))$ is not bounded. Then, there exists a sequence $\left(x_{n}\right)$ of points from $c(\mathbb{R})$ with $\mathrm{d}_{T}\left(O^{\prime}, y_{n}\right) \longrightarrow \infty$, for any $y_{n} \in$ $\pi_{C^{\prime}}\left(x_{n}\right)$. Furthermore, the sequence $\left(x_{n}\right)$ satisfies $\mathrm{d}_{T}\left(O, x_{n}\right) \longrightarrow \infty$, by Theorem 4.2 (2). Passing to further subsequences if need be, we may assume $x_{n}>P^{+}$and $y_{n}>Q^{+}$, say, where $P^{+} \in c(\mathbb{R})$ and $Q^{+} \in c^{\prime}(\mathbb{R})$ are the respective points given by Proposition 4.3 .

We have $\mathrm{d}_{T}\left(x_{n}, y_{n}\right)>\max \left\{\mathrm{d}_{T}\left(O, x_{n}\right), \mathrm{d}_{T}\left(O^{\prime}, y_{n}\right)\right\}$ and, according to Theorem $4.2(1)$, we also have $\operatorname{diam}\left(\pi_{c^{\prime}}\left(B_{\mathrm{d}_{T}\left(x_{n}, c^{\prime}(\mathbb{R})\right)}\left(x_{n}\right)\right)\right) \leq b$, for all $n \in \mathbb{N}$. However, $O \in B_{\mathrm{d}_{T}\left(x_{n}, c^{\prime}(\mathbb{R})\right)}\left(x_{n}\right)$ and so $O^{\prime} \in \pi_{c^{\prime}}\left(B_{\mathrm{d}_{T}\left(x_{n}, c^{\prime}(\mathbb{R})\right)}\left(x_{n}\right)\right)$ for all $n$. From this we deduce $\mathrm{d}_{T}\left(O^{\prime}, y_{n}\right) \leq b$ for all $n$, and this is a contradiction.

Given an arc-length parametrization $c: \mathbb{R} \longrightarrow T(\Sigma)$ of a Teichmüller geodesic and a real number $R>0$, we introduce the subsets

$$
\Pi(c, R)=\left\{x \in T(\Sigma): \pi_{c}(x) \subset c([R, \infty))\right\}
$$

and

$$
\Pi(c,-R)=\left\{x \in T(\Sigma): \pi_{c}(x) \subset c((-\infty,-R])\right\}
$$

of $T(\Sigma)$. We note that, if $R<R^{\prime}$, then $\Pi\left(c, R^{\prime}\right) \subset \Pi(c, R)$ and $\Pi\left(c,-R^{\prime}\right) \subset$ $\Pi(c,-R)$.

Corollary 4.5. For a surface $\Sigma$ and $\epsilon>0$, let $c, c^{\prime}: \mathbb{R} \longrightarrow T(\Sigma)$ be arc-length parametrizations of two $\epsilon$-precompact Teichmüller geodesics. Then, there exists $R>0$ such that the sets $\Pi(c, R), \Pi(c,-R), \Pi\left(c^{\prime}, R\right)$ and $\Pi\left(c^{\prime},-R\right)$ are pairwise disjoint.

Proof. That $\Pi(c, R) \cap \Pi(c,-R)$ and $\Pi\left(c^{\prime}, R\right) \cap \Pi\left(c^{\prime},-R\right)$ are both the empty set for sufficiently large $R$ is a trivial consequence of Minsky's Bounded Projection Theorem. Let us just show $\Pi(c, R) \cap \Pi\left(c^{\prime}, R\right)=\emptyset$, since the remaining cases can 
be proven analogously. We again argue by contradiction, by supposing that for all $n \in \mathbb{N}$ there exists $x_{n} \in \Pi(c, n) \cap \Pi\left(c^{\prime}, n\right)$. Let $y_{n} \in \pi_{c}\left(x_{n}\right)$ and let $z_{n} \in \pi_{c^{\prime}}\left(x_{n}\right)$, for all $n$. Note that, in particular, $\left(y_{n}\right)_{n \in \mathbb{N}}$ and $\left(z_{n}\right)_{n \in \mathbb{N}}$ are unbounded sequences on the geodesics $c(\mathbb{R})$ and $c^{\prime}(\mathbb{R})$, respectively. On passing to subsequences if need be, we have $\mathrm{d}_{T}\left(x_{n}, y_{n}\right) \geq \mathrm{d}_{T}\left(x_{n}, z_{n}\right)$, for all $n$, or $\mathrm{d}_{T}\left(x_{n}, y_{n}\right) \leq \mathrm{d}_{T}\left(x_{n}, z_{n}\right)$, for all $n$. Without loss of generality, we assume the former holds.

The diameter of the projection of $B_{\mathrm{d}_{T}\left(x_{n}, y_{n}\right)}\left(x_{n}\right)$ into $c(\mathbb{R})$ is at most $b$, by Theorem 4.2 (1). In particular, $\mathrm{d}_{T}\left(\pi_{c}\left(z_{n}\right), \pi_{c}\left(x_{n}\right)\right) \leq b$ and it follows $\mathrm{d}_{T}\left(\pi_{c}\left(c^{\prime}\right), y_{n}\right) \leq b$ for all $n \in \mathbb{N}$. This is a contradiction, since $\pi_{c}\left(c^{\prime}\right)$ has bounded diameter, by Corollary 4.4, and $\left(y_{n}\right)$ is an unbounded sequence on the geodesic $c(\mathbb{R})$.

Corollary 4.5 gives us enough information to apply the following lemma, the statement of which is recorded from [4, and deduce sufficiently high powers of $n$ independent pseudo-Anosov mapping classes freely generate a free group of rank $n$.

Lemma 4.6 (Ping-Pong Lemma). Let $X$ be a set and let $f_{1}, \ldots, f_{n}$ be bijections from $X$ to itself. Suppose, for every $i=1, \ldots, n$, there exist pairwise disjoint subsets $A_{1}^{+}, A_{1}^{-}, \ldots, A_{n}^{+}, A_{n}^{-}$of $X$ such that $f_{i}\left(X \backslash A_{i}^{-}\right) \subseteq A_{i}^{+}$and $f_{i}^{-1}\left(X \backslash A_{i}^{+}\right) \subseteq A_{i}^{-}$, for each $i$. Then, under composition, $f_{1}, \ldots, f_{n}$ freely generate a free group of rank $n$.

Corollary 4.7 (11, 16]). For a surface $\Sigma$, let $\phi_{1}, \ldots, \phi_{n}$ be pairwise independent pseudo-Anosov mapping classes in $\operatorname{MCG}(\Sigma)$. Then, there exists a natural number $N$ such that $\phi_{1}^{N}, \ldots, \phi_{n}^{N}$ freely generate a free group of rank $n$.

Proof. Let $c_{i}: \mathbb{R} \longrightarrow T(\Sigma)$ be an arc-length parametrization of the axis of $\phi_{i}$ for each $i=1, \ldots, n$. By Corollary 2.3 there exists a real number $\epsilon>0$ such that $c_{i}$ is $\epsilon$-precompact for each $i=1, \ldots, n$. We note $\epsilon$ depends only on the maximal translation distance among the $\phi_{i}$.

Now Corollary 4.5 applied to all pairs $c_{j}$ and $c_{k}$ of parametrizations for $1 \leq j<$ $k \leq n$ implies there exists $R>0$ such that the sets $\Pi\left(c_{1}, \pm R\right), \ldots, \Pi\left(c_{n}, \pm R\right)$ are all pairwise disjoint. Let $\ell_{\min }>0$ be the minimal translation distance among all pseudo-Anosov mapping classes in $\operatorname{MCG}(\Sigma)$. Let $N$ be the least integer such that $N>2 R / \ell_{\min }$. The mapping classes $\phi_{1}^{N}, \ldots, \phi_{n}^{N}$ and the sets $A_{1}^{ \pm}=\Pi\left(c_{1}, \pm R\right), \ldots$, $A_{n}^{ \pm}=\Pi\left(c_{n}, \pm R\right)$ satisfy the hypotheses of Lemma 4.6. and we conclude $\phi_{1}^{N}, \ldots, \phi_{n}^{N}$ freely generate a free group of rank $n$.

\section{A Quantitative Ping-PONG ARgument For PSeUdo-Anosovs}

The purpose of this section is to give the promised quantitative version of Corollary 4.7. On the way, we will show quantitative versions of Proposition 4.3 and Corollary 4.4 for the axes of pseudo-Anosov mapping classes. Let us begin with the following definition.

Definition 5.1. For a surface $\Sigma, R>0$, and $x \in T(\Sigma)$, we say a curve $\alpha \in \mathcal{S}(\Sigma)$ is $R$-short on $x$ if $\ell_{\alpha}(x) \leq R$, and we let $S_{R}(x)$ be the set of $R$-short curves on $x$.

The next result is a special case of the main result in [1, where Birman-Series show the number of simple closed geodesics on a given surface grows at most polynomially in the length bound. The degree of this polynomial depends only on the topological type of the surface. We note that an improved version of this result has been given by Rivin [21]. 
Theorem 5.2 (1], 21]). For a surface $\Sigma$ and $R>0$, there exists an integer $B=B(R, \Sigma)$ such that the cardinality of the set $S_{R}(x)$ is at most $B$ for all $x \in T(\Sigma)$.

Recall that a pants decomposition for $\Sigma$ is a maximal collection of pairwise distinct and pairwise disjoint curves on $\Sigma$. It is a theorem of Bers that there exists a universal constant $R_{*}=R_{*}(\Sigma)$ such that every point $x \in T(\Sigma)$ has a pants decomposition whose curves each have length at most $R_{*}$ in $x$.

Let $\epsilon>0$ and consider the thick part $T_{\geq \epsilon}(\Sigma)$ of the Teichmüller space of $\Sigma$. A simple area argument shows that, given $\epsilon>0$, there is a constant $R=R(\epsilon)$ such that, for every point $x \in T_{\geq \epsilon}(\Sigma)$, the set $S_{R}(x)$ contains a pants decomposition of $\Sigma$ and a curve transverse to each curve in the pants decomposition. From this discussion, and from Theorem 2.1 and Corollary 2.3, we obtain the following lemma.

Lemma 5.3. For a surface $\Sigma$ and $L>0$, there exists a real number $F=F(L, \Sigma)>$ 0 such that, for any pseudo-Anosov of translation distance at most $L$ and any point $x$ on its axis, the set $S_{F}(x)$ contains a pants decomposition of $\Sigma$ and a curve intersecting each curve in the pants decomposition essentially.

Suppose that $\phi, \psi$ are independent pseudo-Anosov mapping classes of translation distance at most $L>0$, with $c, c^{\prime}: \mathbb{R} \longrightarrow T(\Sigma)$ arc-length parametrizations of their respective axes. By Corollary 2.3, there exists $\epsilon=\epsilon(L, \Sigma)>0$ such that $c(\mathbb{R})$ and $c^{\prime}(\mathbb{R})$ are $\epsilon$-precompact Teichmüller geodesics. By Theorem $4.2(3)$, for any $D>0$ there exists a non-negative real number $M=M(2, D, \epsilon, \Sigma)$ such that any $(2, D)$-quasi-geodesic connecting the ends of an $\epsilon$-precompact geodesic lies in the closed $M$-neighbourhood of the geodesic. Let $F=F(L, \Sigma)$ be the constant given by Lemma 5.3, and recall $B=B\left(e^{2(M+L)} F, \Sigma\right) \geq 0$ is a uniform upper bound on the number of $e^{2(M+L)} F$-short curves over all points in $T(\Sigma)$. Note, $B$ depends only on $D, L$ and $\Sigma$. We have the following result, a quantitative analogue of Proposition 4.3 .

Proposition 5.4. For a surface $\Sigma$, let $\phi, \psi$ be independent pseudo-Anosov mapping classes of translation distance at most $L>0$. Let $c, c^{\prime}: \mathbb{R} \longrightarrow T(\Sigma)$ be arc-length parametrizations of their respective axes so that $O=c(0)$ and $O^{\prime}=c^{\prime}(0)$ together realise the nearest-point distance $D$ of $c(\mathbb{R})$ and $c^{\prime}(\mathbb{R})$. For

$$
R=\max \{B !+2,(B !+2) L\},
$$

the following holds: For all $x \in c(\mathbb{R}) \backslash B_{R}(O)$ and all $y \in c^{\prime}(\mathbb{R}) \backslash B_{R}\left(O^{\prime}\right)$,

$$
\mathrm{d}_{T}(x, y)>\max \left\{\mathrm{d}_{T}(O, x), \mathrm{d}_{T}\left(O^{\prime}, y\right)\right\} .
$$

Proof. Suppose the result were not true. Then, there are points $x \in c(\mathbb{R})$ and $y \in$ $c^{\prime}(\mathbb{R})$ with $\mathrm{d}_{T}(x, O)>R, \mathrm{~d}_{T}\left(y, O^{\prime}\right)>R$ and $\mathrm{d}_{T}(x, y) \leq \max \left\{\mathrm{d}_{T}(O, x), \mathrm{d}_{T}\left(O^{\prime}, y\right)\right\}$. Assume without loss of generality that $\mathrm{d}_{T}(O, x) \geq \mathrm{d}_{T}\left(O^{\prime}, y\right)$. Let $q$ be the concatenation of the unique geodesic segment between $O$ and $O^{\prime}$, the $c^{\prime}(\mathbb{R})$-segment between $O^{\prime}$ and $y$ and the unique geodesic segment between $y$ and $x$. Since $\mathrm{d}_{T}(x, y) \leq \max \left\{\mathrm{d}_{T}(O, x), \mathrm{d}_{T}\left(O^{\prime}, y\right)\right\}$ we have, as in the proof of Proposition 4.3. that $q$ is a $(2, D)$-quasi-geodesic connecting $O$ to $x$. Therefore the image of $q$ is entirely contained in the closed $M$-neighbourhood of the unique geodesic connecting $O$ to $x$, where $M=M(2, D, \epsilon, \Sigma)$ as per Theorem $4.2(3)$.

Let $I=B !+2$. Since the translation distance of $\psi$ is at most $L$ and $R \geq$ $(B !+2) L=I L$, the points $y_{1}=O^{\prime}, y_{2}=\psi\left(O^{\prime}\right) \ldots, y_{I}=\psi^{I-1}\left(O^{\prime}\right)$ all lie on the geodesic from $O^{\prime}$ to $y$, which is a geodesic subpath of the quasi-geodesic $q$. Since the 
image of $q$ is contained in the closed $M$-neighbourhood of the geodesic connecting $O$ to $x$, there are points $x_{1}, \ldots, x_{I}$ in $c(\mathbb{R})$ such that $d\left(x_{i}, y_{i}\right) \leq M$ for all $i=1, \ldots, I$. Since the translation distance of $\phi$ is also at most $L$, it follows that there are points $z_{1}, \ldots, z_{I}$ (not necessarily distinct) such that $d_{T}\left(x_{i}, z_{i}\right) \leq L$ and $z_{i}=\phi^{j(i)}(O)$ for $i=1, \ldots, I$. Therefore $d_{T}\left(y_{i}, z_{i}\right) \leq M+L$ for $i=1, \ldots, I$.

By Lemma 5.3 there exists $F=F(L, \Sigma)>0$ such that the set $S_{F}\left(O^{\prime}\right)$ of short curves in $O^{\prime}$ contains a set $S$ consisting of a pants decomposition and a single curve intersecting each curve in the pants decomposition essentially. Then, every element of the set $S$ is $e^{2(M+L)} F$-short in $O$ by Theorem 3.1. Now, if a curve $\alpha$ is $F$ short in $O^{\prime}$, then $\psi^{i-1}(\alpha)$ is $F$-short in $y_{i}$ for $i=1, \ldots, I$. Therefore $\phi^{-j(i)} \psi^{i-1}(\alpha)$ is $e^{2(M+L)} F$-short in $O$. In particular, all elements of the set $\phi^{-j(i)} \psi^{i-1}(S)$ are $e^{2(M+L)} F$-short in $O$. Moreover, note that the set $\phi^{-j(i)} \psi^{i-1}(S)$ also consists of a pants decomposition and a curve intersecting each curve in the pants decomposition transversally, since $\phi^{-j(i)} \psi^{i-1}$ is a mapping class.

Since the set of $e^{2(M+L)} F$-short curves in $O$ has cardinality at most $B$ and $I>B !+1$, there must be some $k \in\{0, \ldots, I-1\}$ such that $\phi^{-j(k)} \psi^{k-1}(\beta)=\beta$ for all $\beta \in S$. It follows $\phi^{j(k)}$ and $\psi^{k-1}$ share the same action on the set $S(\Sigma)$ of all curves on $\Sigma$. Therefore $\phi^{j(k)}$ and $\psi^{k-1}$ are either equal or, for only a few exceptional surfaces, perhaps differ by a hyperelliptic involution. Regardless, both share common fixed points in $\overline{T(\Sigma)}$ and this is contrary to their independence.

Corollary 5.5. For a surface $\Sigma$ and $L>0$, let $\phi, \psi$ be independent pseudo-Anosov mapping classes of translation distance at most $L>0$. Let $c, c^{\prime}: \mathbb{R} \longrightarrow T(\Sigma)$ be arc-length parametrizations of their respective axes so that $O=c(0)$ and $O^{\prime}=c^{\prime}(0)$ together realise the nearest-point distance of $c(\mathbb{R})$ and $c^{\prime}(\mathbb{R})$. Let $R$ and $b$ be as per Proposition 5.4 and Theorem 4.2, respectively. Then,

$$
\pi_{c}\left(c^{\prime}\right) \subseteq B(O, R+4 b) \cap c(\mathbb{R}) \quad \text { and } \quad \pi_{c^{\prime}}(c) \subseteq B\left(O^{\prime}, R+4 b\right) \cap c^{\prime}(\mathbb{R}) .
$$

Proof. We need only prove one of the inclusions. Suppose, for contradiction, that $\pi_{c^{\prime}}(c)$ is not entirely contained in $B\left(O^{\prime}, R+4 b\right) \cap c^{\prime}(\mathbb{R})$. Then, there are points $x \in c(\mathbb{R})$ and $y \in \pi_{c^{\prime}}(x) \subset c^{\prime}(\mathbb{R})$ with $\mathrm{d}_{T}\left(O^{\prime}, y\right)>R+4 b$. By Theorem 4.2(2), we have

$$
\mathrm{d}_{T}(O, x)+4 b \geq \operatorname{diam}\left(\pi_{c}(O) \cup \pi_{c}(x)\right) \geq \mathrm{d}_{T}\left(O^{\prime}, y\right)>R+4 b
$$

and hence $\mathrm{d}_{T}(O, x)>R$. According to Proposition [5.4, we also have $\mathrm{d}_{T}(x, y)>$ $\mathrm{d}_{T}(O, x)$. In particular, $O \in B_{\mathrm{d}_{T}(x, y)}(x)$. By Theorem4.2(1), $\operatorname{diam}\left(\pi_{c^{\prime}}\left(B_{\mathrm{d}_{T}(x, y)}(x)\right)\right)$ $\leq b$ and so $\mathrm{d}_{T}\left(O^{\prime}, y\right) \leq b$. This is a contradiction, and we deduce the corollary.

Corollary 5.6. For a surface $\Sigma$ and $L>0$, let $\phi, \psi$ be independent pseudo-Anosov mapping classes of translation distance at most $L>0$. Let $c, c^{\prime}: \mathbb{R} \longrightarrow T(\Sigma)$ be arc-length parametrizations of their respective axes so that $O=c(0)$ and $O^{\prime}=c^{\prime}(0)$ together realise the nearest-point distance of $c(\mathbb{R})$ and $c^{\prime}(\mathbb{R})$. Let $R$ and $b$ be as per Proposition 5.4 and Theorem 4.2 , respectively. Then, the sets $\Pi(c, R+6 b)$, $\Pi(c,-R-6 b), \Pi\left(c^{\prime}, R+6 b\right)$ and $\Pi\left(c^{\prime},-R-6 b\right)$ are pairwise disjoint.

Proof. We prove $\Pi(c, R+6 b) \cap \Pi\left(c^{\prime}, R+6 b\right)=\emptyset$. Again, suppose that there exists $x \in T(\Sigma)$ such that $x \in \Pi(c, R+6 b) \cap \Pi\left(c^{\prime}, R+6 b\right)$. Let $y \in \pi_{c}(x), w \in \pi_{c^{\prime}}(y)$ and $z \in \pi_{c^{\prime}}(x)$. Without loss of generality, we assume that $\mathrm{d}_{T}(x, y) \geq \mathrm{d}_{T}(x, z)$. By Theorem 4.2(1) we have $\operatorname{diam}\left(\pi_{c^{\prime}}\left(B_{\mathrm{d}_{T}(x, y)}(x)\right)\right) \leq b$, and in particular $\mathrm{d}_{T}(w, z) \leq b$. 
On the other hand, appealing to Corollary [5.5, we have

$$
\mathrm{d}_{T}(w, z) \geq \mathrm{d}_{T}\left(O^{\prime}, z\right)-\mathrm{d}_{T}\left(O^{\prime}, w\right) \geq R+6 b-R-4 b=2 b>b
$$

and this is a contradiction.

We are ready to give the promised quantitative version of Corollary 4.7 Recall, $\ell_{\min }$ is defined as the minimal translation distance among all pseudo-Anosov mapping classes from $\operatorname{MCG}(\Sigma)$.

Theorem 5.7. For a surface $\Sigma$ and $L>0$, let $\phi_{1}, \ldots, \phi_{n}$ be pseudo-Anosov mapping classes of translation distance at most $L$. Let $R$ and $b$ be as per Proposition 5.4 and Theorem 4.2, respectively. If $N>(2 R+12 b) / \ell_{\min }$, then $\phi_{1}^{N}, \ldots, \phi_{n}^{N}$ freely generate a free group of rank $n$.

Proof. Corollary 5.6 implies the sets $\Pi\left(c_{1}, \pm(R+6 b)\right), \ldots, \Pi\left(c_{n}, \pm(R+6 b)\right)$ are pingpong sets for $\phi_{1}^{N}, \ldots, \phi_{n}^{N}$. The result now follows from the Ping-Pong Lemma.

\section{ACKNOWLEDGEMENTS}

The authors wish to thank Brian Bowditch, Vlad Markovic and Caroline Series for many interesting and helpful conversations. The third author would like to thank the Japan Society for the Promotion of Science and also wishes to thank both Osaka University and the Institut des Hautes Études Scientifiques for their hospitality and for providing a stimulating working environment. The authors wish to thank the referee for his or her careful reading of the manuscript.

\section{REFERENCES}

[1] J. S. Birman, C. M. Series, Geodesics with bounded intersection number on surfaces are sparsely distributed, Topology 24 (1985), no. 2, 217-225. MR793185 (87f:57012)

[2] F. Bonahon, Geodesic laminations on surfaces. Laminations and foliations in dynamics, geometry and topology (Stony Brook, NY, 1998), 1-37, Contemp. Math., 269. MR1810534 (2001m:57023)

[3] B. H. Bowditch, Hyperbolic 3-manifolds and the geometry of the curve complex, in "European Congress of Mathematics, Stockholm, June 27 - July 2, 2004" (ed. A.Laptev) European Mathematical Society Publishing House (2005) 103-115. MR2185739(2006h:57014)

[4] M. R. Bridson, A. Haefliger, Metric spaces of non-positive curvature, Grundlehren der Mathematischen Wissenschaften [Fundamental Principles of Mathematical Sciences], 319, Springer-Verlag, Berlin, 1999. MR1744486 (2000k:53038)

[5] G. D. Daskalopoulos, L. Katzarkov, R. A. Wentworth, Harmonic maps to Teichmüller space. Math. Res. Lett. 7 (2000), no. 1, 133-146. MR1748294 (2001b:32022)

[6] G. D. Daskalopoulos, R. A. Wentworth, Classification of Weil-Petersson isometries, Amer. J. Math. 125 (2003) no. 4, 941-975. MR1993745 (2004d:32011)

[7] B. Farb, L. Mosher, Convex cocompact subgroups of mapping class groups, Geometry and Topology, 6 (2002), 91-152. MR1914566 (2003i:20069)

[8] H. Hamidi-Tehrani, On free subgroups of the mapping class groups, Preprint (1997).

[9] Y. Imayoshi, M. Taniguchi, An introduction to Teichmüller spaces, Springer-Verlag, Tokyo, 1992. xiv+279 pp. MR1215481 (94b:32031)

[10] N. V. Ivanov, Coefficients of expansion of pseudo-Anosov homeomorphisms, translation in $J$. Soviet Math. 52 (1990), no. 1, 2819-2822. MR964259 (89i:32047)

[11] N. V. Ivanov, Subgroups of Teichmüller modular groups, Translations of Mathematical Monographs 115 (1992). MR1195787 (93k:57031)

[12] N. V. Ivanov, Mapping class groups, in "Handbook of geometric topology" (ed. R. Daverman and R. Sher), Elsevier (2001) 523-633. MR1886678 (2003h:57022)

[13] R. P. Kent IV, C. J. Leininger, Shadows of mapping class groups: capturing convex cocompactness, arXiv:math.GT/0505114 (2005). 
[14] H. A. Masur, On a class of geodesics in Teichmüller space. Ann. of Math. (2) 102 (1975), no. 2, 205-221. MR0385173 (52:6038)

[15] H. A. Masur, M. Wolf, Teichmüller space is not Gromov hyperbolic, Ann. Acad. Sci. Fenn. Ser. A I Math. 20 (1995), no. 2, 259-267. MR.1346811 (96f:30048)

[16] J. D. McCarthy, A "Tits-alternative" for subgroups of surface mapping class groups, Trans. Amer. Math. Soc. 291 (1985), no. 2, 583-612. MR800253 (87f:57011)

[17] J. D. McCarthy, A. Papadopoulos, Dynamics on Thurston's sphere of projective measured foliations, Comment. Math. Helv. 64 (1989), no. 1, 133-166. MR982564 (90e:57054)

[18] J. D. McCarthy, A. Papadopoulos, The mapping class group and a theorem of Masur-Wolf. Topology Appl. 96 (1999), no. 1, 75-84. MR1701241 (2000i:32025)

[19] Y. N. Minsky, Quasi-projections in Teichmüller space. J. Reine Angew. Math. 473 (1996), 121-136. MR:1390685 (97b:32020)

[20] R. C. Penner, Bounds on least dilatations, Proc. Amer. Math. Soc. 113 (1991), no. 2, 443450. MR:1068128 (91m:57010)

[21] I. Rivin, Simple curves on surfaces. Geom. Dedicata 87 (2001), no. 1-3, 345-360. MR1866856 (2003c:57018)

[22] H. L. Royden, Automorphisms and isometries of Teichmüller space. 1971, Advances in the Theory of Riemann Surfaces, Ann. of Math. Studies, No. 66. Princeton Univ. Press, Princeton, N.J. MR0288254 (44:5452)

[23] W. P. Thurston, Three dimensional manifolds, Kleinian groups and hyperbolic geometry, Bull. Amer. Math. Soc. (N.S.) 6 (1982), no. 3, 357-381. MR648524 (83h:57019)

[24] S. A. Wolpert, The length spectra as moduli for compact Riemann surfaces. Ann. of Math. (2) 109 (1979), no. 2, 323-351. MR528966 (80j:58067)

[25] S. A. Wolpert, Geometry of the Weil-Petersson completion of Teichmüller space. Surveys in differential geometry, Vol. VIII (Boston, MA, 2002), 357-393. MR2039996 (2005h:32032)

School of Mathematics, University of Southampton, Southampton SO17 1BJ, EngLAND

E-mail address: j.w.anderson@soton.ac.uk

Mathematics Institute, University of Warwick, Coventry CV4 7AL, England

E-mail address: jaram@maths.warwick.ac.uk

Institut des Hautes Études Scientifiques, Le Bois-Marie, 35 Route de Chartres, F91440 Bures-Sur-Yvette, France

Current address: Department of Mathematical and Computing Sciences, Tokyo Institute of Technology, 2-12-1 O-okayama, Meguro-ku, Tokyo 152-8552, Japan

E-mail address: kjs2006@alumni.soton.ac.uk; shackleton.k.aa@m.titech.ac.jp 